
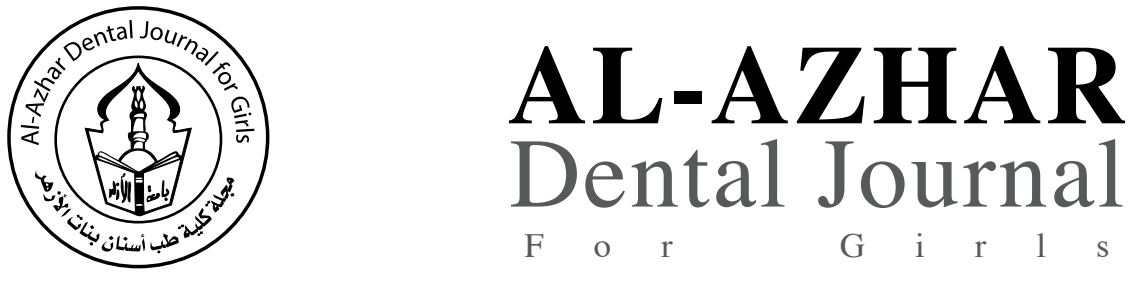

The Official Publication of The Faculty of Dental Medicine For Girls, Al-Azhar University Cairo, Egypt.

ADJ-for Grils, Vol. 4, No. 1, January (2017) - PP. 57:62

\title{
The Effects of Two Different Palatal Expanders on Nasal and Pharyngeal Airway Volumes as Measured by CBCT
}

\author{
Amira H. Allam ${ }^{(1)}$, Tarek H. Marei ${ }^{(2)}$, Fatma A. Abd Elsamad ${ }^{(2)}$ and Sayed M. Mekheimar ${ }^{(3)}$
}

Codex : 08/1701

dentaljournal.forgirls@yahoo.com

\section{KEYWORDS}

Palatal expanders, airway, nasal, pharyngeal, cbct

\begin{abstract}
Objective: To evaluate and compare the effect of memory palatal split screw (Memorax) to that of conventional Hyrax rapid palatal expanders on nasal and pharyngeal airway volumes, which were measured and recorded by Cone beam computed tomography (CBCT). Materials and Methods: This study was conducted over 14 subjects of adolescent patients with bilaterally constricted maxillary arches, the sample contained 12 females and 2 males, with age ranged from $12 \mathrm{ys}$ to $15 \mathrm{ys}$, with a mean of $13.6 \pm 1.4$. The sample was divided into two age matched groups, the Memorax group $(\mathrm{N}=7)$ subjects with a mean age of $13.5 \pm 0.4$, received the memory palatal split screw appliance (Memorax). The Hyrax group ( $\mathrm{N}=7$ ) subjects with a mean of $13.4 \pm 1$ were received the conventional Hyrax palatal expander. For all subjects taking part in the study, maxillofacial CBCTs and NOSE questionnaire records were taken before expansion $\left(\mathbf{T}_{1}\right)$ and after 3 months at the time of removal of the expanders $\left(\mathbf{T}_{2}\right)$. Data were explored for normality using Kolmogorov-Smirnov test of normality. Paired t test was used to compare mean values of before and after treatment. Independent (unpaired) test was used to compare between the two groups. Results: Memorax showed a significant increase in nasal airway volume as well as naso- , palato- and total pharyngeal airway volume. While Hyrax showed a non- significant decrease in the palatopharyngeal airway volume. Both groups showed a non- significant difference in the glossopharyngeal airway volume as well as in the NOSE questionnaire results. Conclusion: Memorax could be used in patients suffered from symptomatic nasal obstruction due to bilateral maxillary constriction, in order to improve their breathing as a primary purpose as well as treating malocclusion. It also may be advantageous because it shortens the maxillary expansion period, provides additional expansion in the retention period and generates light forces relative to the conventional Hyrax screw.
\end{abstract}

The paper was extracted from master research study at Faculty of dental medicine for girls, Al-Azhar University

1. BDS 2002 Ain Shams University- A resident in Master Degree program, Orthodontic Department, Faculty of dental medicine for girls, Al-Azhar University.

2. Assistant Professor, Orthodontic Department -Faculty of Dental Medicine for girls Al-Azhar University

3. Professor of ENT, Head of ENT Department -Faculty of Medicine for girls- Al-Azhar University 


\section{INTRODUCTION}

Maxillary constriction is associated with several problems that include cross bite (dental and/or skeletal), occlusal disharmony, esthetics and functional problems such as narrowing of the pharyngeal airway. It may also play a role in the etiology of obstructive sleep apnea (OSA), a condition characterized by the episodic cessation of breathing during sleep.

Rapid maxillary expansion (RME) is a welldocumented orthodontic treatment modality for correcting transverse maxillary deficiency. It was first introduced by Angell in the 19th century, ${ }^{(1)}$ and it was suggested as a treatment for respiratory disturbances and cited for its effects over the maxilla. Over the years, the method described by Angell was attempted with varying success, and it was finally reintroduced by Haas, who suggested that the application of RME created considerable changes in the nasomaxillary complex. ${ }^{(2)}$

A new maxillary memory palatal split screw discussed recently, that includes nickel-titanium open coil springs in the screw bed in order to lessen massive expansion forces. This new screw could produce rapid, constant, and physiological expansion forces, thus making the expansion procedure more effective, more physiological, and well tolerated by patients.

Although studies about memory screw have increased in the past years, till date no study has been carried out to compare the effects of RME caused by memory screw and Hyrax screw on the dento-facial structures and airway dimensions. The available Studies have used a lot of technologies to evaluate the effects of RME over nasal cavity surface area. However most of them have not taken into account the effects on nasal volume and pharyngeal airway volume as well.

\section{MATERIALS AND METHODS}

This study was conducted over 14 subjects of adolescent patients with bilaterally constricted maxillary arches, the sample contained 12 females and 2 males, with age ranged from $12 \mathrm{ys}$ to $15 \mathrm{ys}$, with a mean of $13.6 \pm 1.4$. The sample was divided into two groups, the Memorax group $(\mathrm{N}=7)$ subjects with a mean age of $13.5 \pm 0.4$, received the memory palatal split screw appliance (Memorax). The Hyrax group $(\mathrm{N}=7)$ subjects with a mean of $13.4 \pm 1$ were received the conventional Hyrax palatal expander.

The activation of the Memorax, was 6 quarter turns per day; twice in the morning, twice in midday, and twice at the evening, $(0.2 \mathrm{~mm}$ per quarter turn) (1.2 mm per day), with total number of quarter turns ranged from 36 to 48 turns, with mean of 42 , which correspond to $8.4 \mathrm{~mm}$ expansion, the time elapsed till achieving over correction ranged from 6 to 8 days. With a mean of 7 days. While In Hyrax group, Expansion of Hyrax was at a rate of 2 quarter turns per day; once in the morning and once at evening ( $0.2 \mathrm{~mm}$ per turn) $(0.4 \mathrm{~mm}$ per day), with total number of turns ranged from 38 to 46 turns, with mean of 44 , which correspond to $8.8 \mathrm{~mm}$ expansion, the time elapsed till achieving over correction was from 19 to 23 days. With a mean of 22 days. (Fig 1)

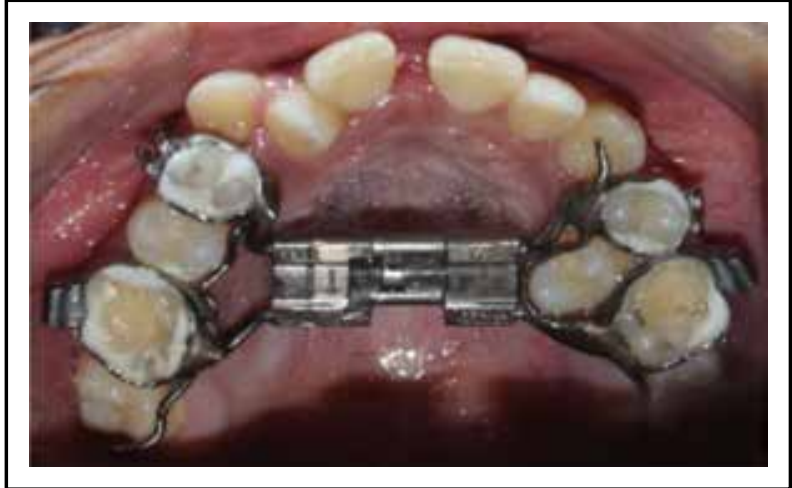

Fig. (1) Memorax during active Expansion period 
For all subjects taking part in the study, maxillofacial CBCTs and NOSE questionnaire records were taken before expansion $\left(\mathbf{T}_{\mathbf{1}}\right)$ and after 3 months at the time of removal of the expanders $\left(\mathbf{T}_{2}\right)$. Nasal airway volume using Mimics software, was measured at T1 and T2, nasal space was calculated at the pre and post-operative scans. Software was adjusted at certain threshold for recording airspaces for all scans. At the coronal views, Space of Nasal cavity was then drawn using the assigned threshold starting from the external naris anteriorly, till the last coronal before sphenoid air sinus appeared. The drawn threshold was then automatically calculated as the nasal cavity space. While pharyngeal airway volume, was segmented into 3 volumes to be measured separately, Nasopharynx (Vn), Palatopharynx $(\mathrm{Vp})$, Glossopharynx $(\mathrm{Vg})$, and their sum which is the Total pharynx $(\mathrm{Vt})$. They were measured using Dolphin 3D program airway tool.

\section{RESULTS}

Results revealed that the nasal airway volume significantly increased after treatment in both the Memorax $(p=0.00099)$ and Hyrax $(p=0.0014)$ groups. Regarding the percent change, a greater percent change was noted in the Memorax group which was statistically significant ( $\mathrm{p}=0.0368)$. (Fig 2)

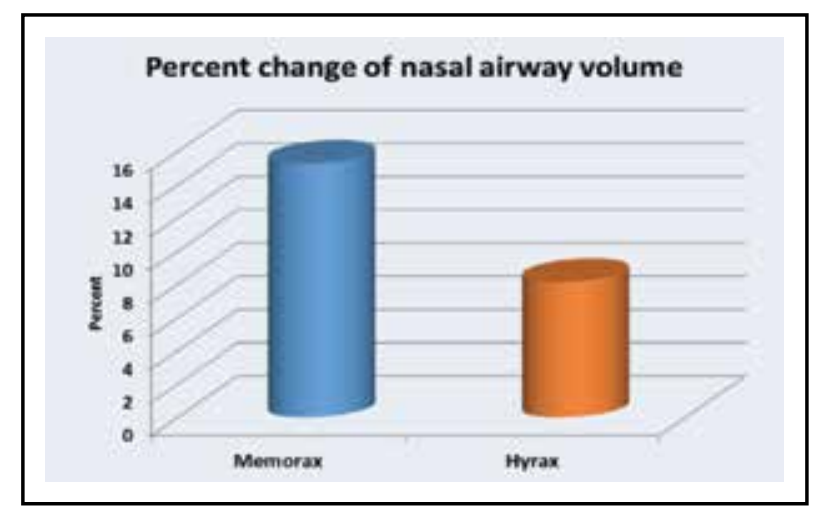

Fig. (2)

In the Memorax group, pharyngeal airway volume significantly increased after treatment in each compartment and in the total volume, While In the Hyrax group, the volume of the Nasopharynx, Glossopharynx and the total pharyngeal airway volume significantly increased, however the Palatopharyngeal volume showed a non-significant decrease in volume after treatment $(\mathrm{p}=0.1303)$. (Fig 3)

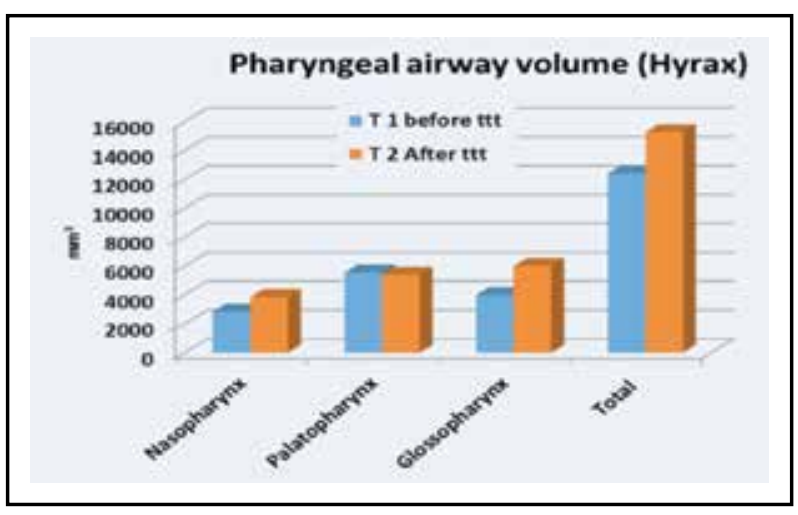

Fig. (3)

Patients fill a questionnaire at $\mathbf{T 1}$ and other one at $\mathbf{T 2}$, each questionnaire has a score range from 0 to 100 . The nearer to the 0 score are the best results, the nearer to 100 score are the worst results. Paired t test revealed that the questionnaire score significantly decreased after treatment in both the Memorax $(\mathrm{p}<0.0001)$ and Hyrax $(\mathrm{p}<0.0001)$ groups but Comparing the pre-treatment and post-treatment values of both expanders, unpaired $t$ test revealed no significant difference, while Regarding the percent change, a greater percent non-significant decrease was noted in the Hyrax group ( $p=0.7898)$. (Fig 4)

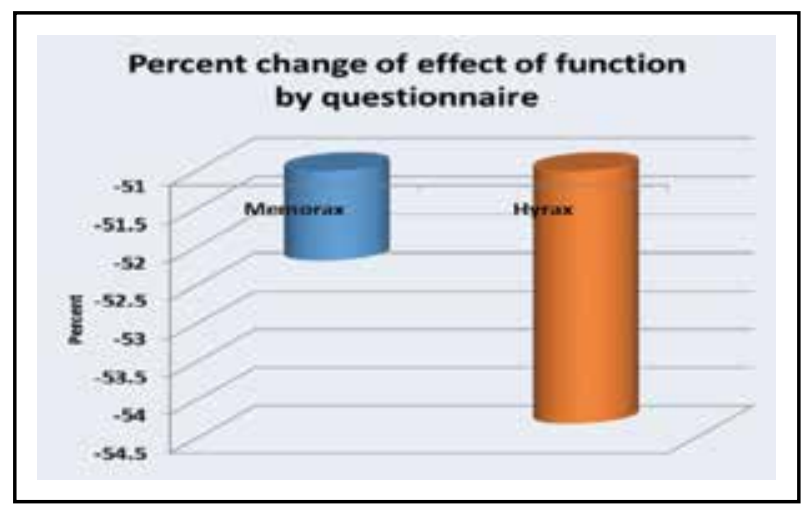

Fig. (4) 


\section{DISCUSSION}

The nasal airway volume significantly increased after treatment in both the Memorax and Hyrax groups, which agrees with many studies discussed Maxillary expansion effects on nasal cavity, as it affected the palatal suture and demonstrated anterior and posterior skeletal widening of the nasal cavity, with corresponding soft tissue changes and increased airway volume. ${ }^{(3-6)}$

Other in-vitro studies revealed the same results regarding nasal cavity widening specially the anterior and inferior parts. ${ }^{(7-9)}$ Also the nasopharynx has expanded significantly as a result of inferio-anterior displacement of palatal shelves. ${ }^{(10)}$ The multiple backward regressions showed that the more the subjects presented a reduced nasal volume in the middle and lower compartments, the more they would benefit from RME as it produces a functional improvement of the breathing pattern in patients with nasal obstruction or stenosis. ${ }^{(11)}$

For Pharyngeal airway volume, previous studies divided the airway into various compartments evaluating volume before and after RME to better clarify the effects of treatment. Several authors have reported a significant enlargement of the nasal cavity and nasopharynx, in agreement with the present results, but no significant increase in the other investigated airway compartments, such as the palatopharynx and glosso-pharynx, suggesting that effects on the upper airway were local as a result of soft tissue adaptation farther from the mid-palatal suture $^{(11)}$, while according to the present results, not only the nasal and nasopharyngeal airways but also the glossopharyngeal airway compartment underwent significant volume increases in the post treatment records by both appliances, which agrees with the findings of Iwasaki et al in 2013 who reported that tongue posture changes as a secondary effect of RME, which increased the retropharyngeal space and improved breathing in children with nasal obstructions. ${ }^{(12)}$
On the contrary. According to the results of our study, in the Memorax group, there was a good response from the respiratory mucosal tissue, which seemed to follow the bony expansion as a respond to the forces applied from the appliances. However in the Hyrax group, the volume of the nasopharynx, glosso-pharynx and the total pharyngeal airway volume significantly increased after treatment. While, the palatopharyngeal volume showed a non-significant decrease after treatment which agrees with the results of Smith who found that the oropharyngeal airway volume decreased a little which was not significant and the author attributed it to the lowering of the palatine vault. ${ }^{(13)}$ Also zeng in 2013 agreed with this non-significant decrease in the oropharynx, relating that to three reasons; Firstly, the breathing stage of the patients which was difficult to control. Secondly, there was a large variance among the sample. Last but not least, the average expansion amount was about not large enough to expand the pharyngeal airway according to the procedures of his study. ${ }^{(14)}$

A recent study by Almuzian et al 2016 which investigated the effect of hyrax on upper and lower retropalatal spaces, revealed that The upper retropalatal space has reduced significantly which might be due to inferio-anterior displacement of palatal shelves secondary to RME, with subsequent stretch of palate-pharyngeus muscle leading to anterior displacement of the posterior pharyngeal wall, or due to trauma of the retropalatal tissue following midpalatine suture split. ${ }^{(10)}$

Nevertheless, differences in compartmental segmentation were noticeable between the present method and the other-mentioned studies, especially those divided pharynx into upper and oropharyngeal airway, which might explain the different outcomes. ${ }^{(15-18)}$

On the other hand, Memorax showed significantly increased volumes of nasal airway as well as nasopharynx, palatopharynx, and total volume compared to that of hyrax as the screw produces rapid 
and constant physiological expansion. Thus, these forces result in more effective maxillary expansion in a shorter duration. Also it may be due to the further expansion produced by the nickel titanium coil spring during the retention period. A study has stressed that maxillary expansion induced by low physiological forces followed by rapid separation of the mid-palatal suture stimulates adaptation of the processes of the nasomaxillary structures and results in less relapse potential in the retention period. (19) Isaacson and Ingram in 1964 hypothesized that the total expansion might be physiologically stable in a shorter net treatment time with expansion procedures carried out at lower forces. ${ }^{(20)}$

There was no significant difference between Memorax and Hyrax groups in the Glossopharynx volume, which is the compartment that showed the highest errors percentage in our study, as it was the most affected by the difficulty to reposition the tongue (due to change in tongue posture), ${ }^{(12)}$ the mandible (as a result of the open bite due to immature contact occur secondary to RME ) and the difficulty to hold breath during CBCT scanning, even though these evidences are still questionable, especially in long-term, follow-up studies. ${ }^{(11)}$

Nose questionnaire results showed a significant difference between pre and post treatment of both groups, which seemed to be equal $(p<0.0001)$ as the patients in both groups were able to differentiate between their symptoms in the pre and post treatment periods. On the other hand, statistical analysis showed no significant difference between both groups, and this was mainly due to the sample size, as well as the patients young ages which might were confused by the questions. Also some studies showed that perception can vary considerably and correlation between objective and subjective findings were often contradictory. ${ }^{(21-25)}$

\section{CONCLUSIONS}

1. Memorax appliance produced faster palatal expansion than Hyrax, and it was more tolerated by most of patients in spite of the higher rate of screw opening during expansion.

2. The Memorax group showed a more significant increase in nasal and pharyngeal airway volumes after RME compared to that of the conventional Hyrax group.

3. Hyrax group showed a nonsignificant decrease in the palatopharyngeal airway volume after RME.

4. The Nose questionnaire was a useless tool to indicate the difference between both appliances in the improvement of breathing function.

\section{RECOMMENDATIONS}

1. More studies on the dentoskeletal effects of the Memorax expander are needed.

2. Further investigations are needed to analyze the forces and stresses produced by the Memorax in both active expansion and retention periods.

3. Arabic version of the NOSE questionnaire will be beneficial in further studies about airway improvement after different orthodontic, orthognathic, and even otolaryngologic interventions

4. Combining morphological records with functional respiratory analysis using a specific functional measurement devices such as; Acoustic rhinometry, Rhinomanometry, and polysomnography, is therefore recommended in further investigations dealing with improvement of breathing function as a resultant of RME.

\section{REFERENCES}

1. Angell EC. Treatment of irregularities of the permanent or adult teeth. Dental Cosmos. 1860; 1: 540-44. Cited from: Hakan El, and Palomo JM. Three-dimensional evaluation of upper airway following rapid maxillary expansion, A CBCT study. Angle Orthod. 2014; 84: 265- 73.

2. Haas AJ. Rapid expansion of the maxillary dental arch and nasal cavity by opening the mid palatal suture. Angle Orthod. 1961; 31: 73-89. 
3. Pangrazio-Kulbersh V, Wine P, Haughey M, Pajtas B, and Kaczynski R. Cone beam computed tomography evaluation of changes in the naso-maxillary complex associated with two types of maxillary expanders. Angle Orthod, 2012; 82: 448- 57.

4. Emirzeoglu M, Sahin B, Celebi M, Uzun A, Bilgic S, and Tontus HO. Estimation of nasal cavity and conchae volumes by stereological method. Folia Morphol, 2012; 71: $105-8$.

5. Zeng J, and Gao X. A prospective CBCT study of upper airway changes after rapid maxillary expansion. Int J Pedi Otorhinolaryngo, 2013; 77: 1805-10.

6. Kanomi R, Deguchi T, Kakuno E, Yamamoto T, and Roberts W. CBCT of skeletal changes following rapid maxillary expansion to increase arch-length with a development-dependent bonded or banded appliance. Angle Orthod, 2013; 83: 851-7.

7. Marei TH. Biomechanical effects of rapid maxillary expansion on the craniomaxillary complex: A finite element study. M. Sc. Thesis. 1998.

8. Marei T, Sayed H, Ibrahim S and Abdel- Kader HM Respiratory efficiency and rapid maxillary expansion (RME): A finite element study. AlAzhar J Dent Science, 2001; 4: 367.

9. Marei T, Ibrahim S and Abdel- Kader HM. Changes in nasal airway subsequent to rapid maxillary expansion: A finite element study. AlAzhar J Dent Science, 1999; 2: 25.

10. Almuzian M, Ju X, Almukhtar A, Ayoub A, Al-Muzian L, and McDonald JP. Does rapid maxillary expansion affect nasopharyngeal airway? A prospective Cone Beam Computerised Tomography (CBCT) based study. j.surge, 2016; 666X: 00125-0

11. Fastuca R, Perinetti G, Zecca PA, Nucera R, and Caprioglio A. Airway compartments volume and oxygen saturation changes after rapid maxillary expansion: A longitudinal correlation study. Angle Orthod, 2015; 85: 955- 61.

12. Iwasaki $\mathrm{T}$, et al. Tongue posture improvement and pharyngeal airway enlargement as secondary effects of rapid maxillary expansion: A cone-beam computed tomography study. Am J Orthod Dentofacial Orthop, 2013; 143:235-45.

13. Smith T, Ghoneima A, Stewart K, Liu S, Eckert G, Halum $\mathrm{S}$, et al. Three dimensional computed tomography analysis of airway volume changes after rapid maxillary expansion. Am J Orthod Dentofac Orthop, 2012; 141: 618-26.

14. Zeng J, and Gao X. A prospective CBCT study of upper airway changes after rapid maxillary expansion. Int J Pedi Otorhinolaryngo, 2013; 77: 1805-10.

15. Hakan El, and Palomo JM. Three-dimensional evaluation of upper airway following rapid maxillary expansion A CBCT study. Angle Orthod. 2014; 84:265-73.

16. Caprioglio A, et al. Rapid maxillary expansion in growing patients: Correspondence between 3-dimensional airway changes and polysomnography. Int J Pedi Otorhinolaryngo, 2014: 23-2724.

17. Rana SS, Duggal R, and Kharbanda OP. Area and volume of the pharyngeal airway in surgically treated unilateral cleft lip and palate patient: A cone beam computed tomography study. J Cleft Lip Palate and Craniofac Anom, 2015; 2 : 52-68.

18. Fastuca R, Perinetti G,Zecca PA, Nucera R, and Caprioglio A. Airway compartments volume and oxygen saturation changes after rapid maxillary expansion: A longitudinal correlation study. Angle Orthod, 2015; 85: 955- 61.

19. Halicioğlu K, Kiliç N, Yavuz I and Aktan B. Effects of rapid maxillary expansion with a memory palatal split screw on the morphology of the maxillary dental arch and nasal airway resistance. Eu J Orthod, 2010; 32: 716-20.

20. Isaacson RJ, and Ingram AH. Forces produced by rapid maxillary expansion. II. Forces present during treatment. Angle Orthod, 1964; 34: 261-70

21. Cole P. Stability of nasal airflow resistance. Clin Otolaryngol Allied Sc, 1989; 14:177-82.

22. Semeraro A and De Colle W. Computerized rhinomanometry in legal medicine, Acta Otorhinolaryngol Ital, 1989; 9: 413-20.

23. Fairley J, Durham LH and Ell SR. Correlation of subjective sensation of nasal patency with nasal inspiratory peak flow rate, Clin Otolaryngol Allied Sci, 1993; 18: 19-22.

24. Roithmann R, et al. Acoustic rhinometry, rhinomanometry, and the sensation of nasal patency: a correlative study. $\mathrm{J}$ Otolaryngol, 1994; 23: 454-8.

25. Wang DY, et al. Acoustic rhinometry in nasal allergen challenge study: which dimensional measures are meaningful? Clin Exp Allergy, 2004; 34: 1093-8. 